\title{
Edge Detection in Remote Sensing Images Based on Fuzzy Image Representation
}

\author{
E.V. Pugin ${ }^{1}$, A.L. Zhiznyakov ${ }^{1}$ \\ ${ }^{1}$ Vladimir State University named after Alexander and Nikolay Stoletovs, Gorky Street 87, Vladimir, Russia
}

\begin{abstract}
Edge detection is an important task in image processing. There are a lot of approaches in this area: Sobel, Canny operators and others. One of the perspective techniques in image processing is the use of fuzzy logic and fuzzy sets theory. They allow us to increase processing quality by representing information in its fuzzy form. Most of the existing fuzzy image processing methods switch to fuzzy sets on very late stages, so this leads to some useful information loss. In this paper a novel method of edge detection based on fuzzy image representation and fuzzy pixels is proposed. With this approach we convert the image to fuzzy form on the first step. Different approaches to this conversion are described. Several membership functions for fuzzy pixel description and requirements for their form and view are given. A novel approach to edge detection based on Sobel operator and fuzzy image representation is proposed. Experimental testing of developed method was performed on remote sensing images. Comparison of result with Sobel, Prewitt, Roberts and Canny operators is presented. Developed method selected more details (edges) rather then Sobel, Prewitt and Roberts operators, but less than Canny operator. This is because the selected convolution kernel (Sobel) has size $3 \times 3$. There are also used only simple functions of estimating the real intensities of pixels. Later, to increase quality it is necessary to use more complex masks of size $5 \times 5$ and $7 \times 7$ or median filters. Developed approach showed its workability in solving image processing problems. The proposed fuzzy model in the future can be extended to use higher level fuzzy sets (Type-2 FS and others).
\end{abstract}

Keywords: edge detection; fuzzy features; fuzzy image representation; fuzzy sets

\section{Introduction}

To extract information from remote sensing image different methods of image processing are used. Edge detection is one of these methods. It can be used to further extraction of interesting objects. There are a lot of algorithms developed in this area. The most popular are Canny, Sobel, Prewitt, Roberts operators and some others $[1,2,3]$. One of the perspective techniques in image processing is the use of fuzzy logic and fuzzy sets theory $[4,5,6]$. Different algorithms and methods use different approaches to fuzzy processing. Linguistic variables are useful when the results are well distinguishable [7, 8, 9], but this is not very common situation. On the other hand it is better to process the results using analytical methods. Most papers suggest switching to fuzzy sets on very late stages of processing when the source image is enhanced, converted or somehow preprocessed. Papers that extract fuzzy properties based on existing crisp features can be taken to this category $[10,11]$. Early defuzzification of the results has also negative influence on fuzzy processing [12]. All these things lower flexibility of fuzzy approach.

Other drawback is the use of fuzzy sets of first type (Type-1 Fuzzy Sets or T1FS), that have very little amount of uncertainty. To solve this issue type-2 fuzzy sets (T2FS) and other more high-type fuzzy sets were introduced [13, 14]. Also, other types of sets based on fuzzy sets are evolving: rough sets, soft sets, soft rough sets, blurry sets and others [15, 16].

In this paper we propose a novel approach to image processing based on fuzzy sets, that suggest a transition to fuzzy image representation with fuzzy pixels on the earliest stages of processing. With this approach all extracted features are fuzzy by definition and defuzzification process at best should be done only when retrieving information from the computer system.

\section{Fuzzy image representation}

Continuous image can be described as two-dimensional signal $f(x, y)$, where $x$ and $y$-coordinates. During formation of digital image transition to discrete coordinates and values of intensities are performed:

$$
F(x, y)=D[f(x, y)]
$$

where $D[\cdot]$ - transformation operator from continuous signal to discrete, that is implemented on hardware, $F(x, y)-$ the discrete image. Obviously that with insufficient level of quantization, signal levels are rounded to integers. Then real intensity level of point $R(x, y)$ could be computed as

$$
R(x, y)=F(x, y)+d(x, y),
$$

where $d(x, y)$ - round error. More noticeable distortion is brought by different noises $v(x, y)$, that could be greater than 1 . We will use the simplest noise model - additive noise. More complex models of noise could be investigated accordingly. Then the real intensity level could be calculated as

$$
R(x, y)=F(x, y)+d(x, y)+v(x, y)
$$




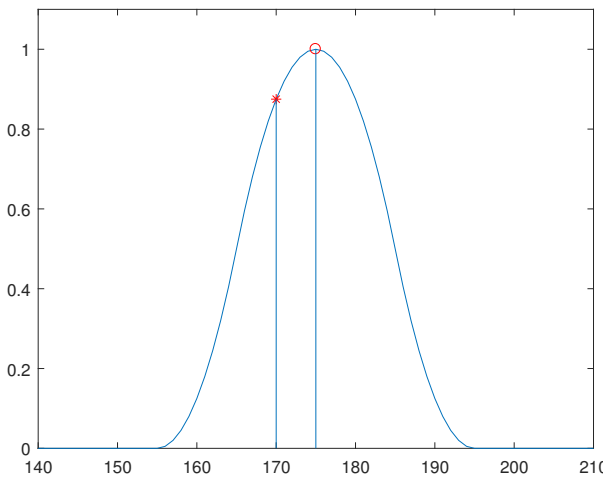

(a)

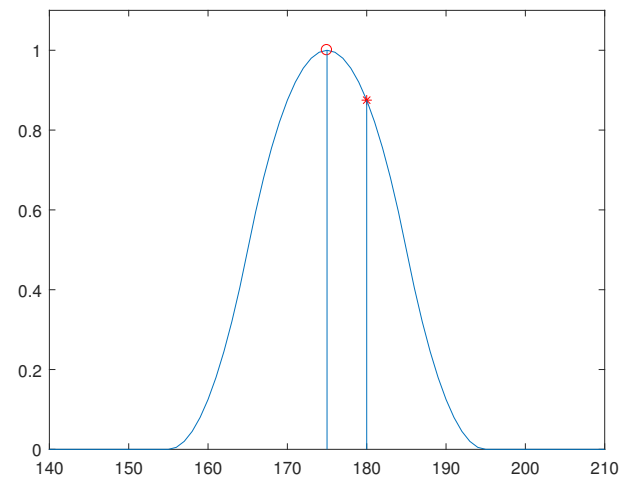

(b)

Fig. 1. Membership function of image fuzzy pixel. Real intensity level at $\mu(F(x, y))=1$ : a) greater, b) less than current.

Computations that does not take into account these details, soon could accumulate big error related to source continuous image. Methods of fuzzy sets theory allow us to save the uncertainty till the latest stages of image processing and analysis. To do it we should switch to fuzzy image representation. $U(F, x, y)$

$$
U(F, x, y)=\mu(F(x, y)),
$$

where $\mu(F(x, y))$ - membership function of a pixel with coordinates $(x, y)$ to intensity level $F(x, y)$. Graphically this can be represented as shown on Fig. 1.

There are a lot of membership functions known. One must select those which satisfy the following conditions

$$
\begin{gathered}
\lim _{l \rightarrow \infty} \mu(F(x, y))=0, \\
\int_{0}^{L-1} \mu(F(x, y)) d l>0, \quad l \in[0 ; L-1] .
\end{gathered}
$$

These include triangular, trapezoidal, bell, Gauss-like, $\pi$ functions and others. In the simplest case we will be using $\pi$-function which is based on $s$-function:

$$
\begin{gathered}
\pi(l)= \begin{cases}s\left(l, c-b, c-\frac{b}{2}, c\right), & l \leq c, \\
1-s\left(l, c, c+\frac{b}{2}, c+b\right), & l \geq c,\end{cases} \\
s(l)= \begin{cases}0, & l \leq a, \\
2\left(\frac{l-a}{c-a}\right)^{2}, & a \leq l \leq b, \\
1-2\left(\frac{l-c}{c-a}\right)^{2}, & b \leq l \leq c, \\
1, & l \geq c,\end{cases}
\end{gathered}
$$

where $b=\frac{a+c}{2}, l$-intensity level. Form of $\pi$-function is shown on Fig. 1 .

To pick $\mu(F(x, y))=\pi(F(x, y))$ function, that is, pick $b$ and $c$ parameters, it is necessary to extract some additional information from the image. Firstly, let us simplify this task reducing the selection to single parameter $I_{c}$ - center of membership function $\pi\left(I_{c}\right)=1$. In this case $\pi$-function lies symmetrically on this point. To set slope inclination, we must choose necessary width of $w$ section, where $\pi(x)>0$. Then let us use the following equation to find parameters of function $\pi$ :

$$
b=\frac{w}{2}, \quad c=I_{c} .
$$

Value of $w$ is chosen empirically, e.g. $w=60$. With small value of $w$ slope will be very big, and small intensity deviations will make the pixel insignificant $(\pi<0.5)$.

Parameter $I_{c}$ can be selected differently. In our case $\pi\left(I_{c}\right)=1$ means real intensity of the pixel, and $\pi(F(x, y)) \neq 1$ shows interference, noise, errors in quantization and similar errors. Value of $I_{c}$ can be computed using neighbor of point $(x, y)$, that is shown on Fig. 2. Let us consider some possible approaches:

1. average between horizontal pixels

$$
I_{c}=\left(P_{4}+P_{8}\right) / 2
$$

2. average between vertical pixels

$$
I_{c}=\left(P_{2}+P_{6}\right) / 2 \text {, }
$$

3. average in 4-neighbour $D_{4}$

$$
I_{c}=\left(P_{2}+P_{4}+P_{6}+P_{8}\right) / 4,
$$




\begin{tabular}{|l|l|l|}
\hline$P_{1}$ & $P_{2}$ & $P_{3}$ \\
\hline$P_{8}$ & $P_{0}$ & $P_{4}$ \\
\hline$P_{7}$ & $P_{6}$ & $P_{5}$ \\
\hline
\end{tabular}

Fig. 2. Neighbour of pixel $P_{0}$ with coordinates $(x, y)$.

\begin{tabular}{|c|c|c|}
\hline-1 & -2 & -1 \\
\hline 0 & 0 & 0 \\
\hline 1 & 2 & 1 \\
\hline
\end{tabular}

(a)

\begin{tabular}{|l|l|l|}
\hline-1 & 0 & 1 \\
\hline-2 & 0 & 2 \\
\hline-1 & 0 & 1 \\
\hline
\end{tabular}

(b)

Fig. 3. Sobel kernels: a) horizontal, b) vertical.

4. average in 8 -neighbour $D_{8}$

$$
I_{c}=\frac{1}{8} \sum_{i=1}^{8} P_{i},
$$

5. average in d-neighbour $D_{d}$

$$
I_{c}=\left(P_{1}+P_{3}+P_{5}+P_{7}\right) / 4,
$$

In more complex case to get $I_{c}$ value one could use one of the existing smoothing methods like median filters, approximations etc.

\section{Edge detection}

Most of the edge detection operators uses gradient operator that has modulo $|\nabla G|$ and direction $\theta$

$$
\begin{gathered}
|\nabla G|=\sqrt{G_{x}^{2}+G_{y}^{2}}, \\
\theta=\arctan \frac{G_{y}}{G_{x}},
\end{gathered}
$$

where $G_{x}=M_{x} * G, G_{y}=M_{y} * G-$ the result of convolution operator with horizontal and vertical matrices.

Let us consider possibility of the use of fuzzy pixels in Sobel and Prewitt edge detection operator (Fig. 3, 4). To do this we must process the membership function values $\pi(F(x, y))$ in addition to normal intensity levels. Calculations of $G_{x \mu}$ and $G_{y \mu}$ can be done analogously. The difference here is in gradient computations of $\pi$-function.

$$
|\nabla \pi|=1-\sqrt{G_{x \mu}^{2}+G_{y \mu}^{2}}
$$

We take complementary value because after squaring, sum and square root operations from values on interval [0; 1$]$, the result is near to 0 . Final value of gradient will be

$$
\left|\nabla G_{\pi}\right|=|\nabla G||\nabla \pi| .
$$

Threshold value of gradient can be selected manually or with different binarization techniques (e.g. with Otsu method [17]).

\begin{tabular}{|c|c|c|}
\hline-1 & -1 & -1 \\
\hline 0 & 0 & 0 \\
\hline 1 & 1 & 1 \\
\hline
\end{tabular}

(a)

\begin{tabular}{|l|l|l|}
\hline-1 & 0 & 1 \\
\hline-1 & 0 & 1 \\
\hline-1 & 0 & 1 \\
\hline
\end{tabular}

(b)

Fig. 4. Prewitt kernels: a) horizontal, b) vertical. 


\section{Testing}

Let us consider transition to fuzzy pixels. Test remote sensing image has size $160 \times 160$. Simple averaging procedures described above were applied to it. Result images are shown on Fig. 5, according membership functions are shown on Fig. 6 and the values are put in Table 1. Comparison of different methods is shown on Fig. 7.

Proposed method extracted more details (edges) than Sobel, Prewitt and Roberts operators, but less than Canny operator. This is because the selected convolution kernel (Sobel) has size 3x3. Also we used only simple functions during real pixel intensity estimation. To increase quality of the results more complex masks $(5 \times 5,7 \times 7)$ could be used and median filters also.

On processed images we can see that proposed method found a lot of "islands", which in the original image are green plantings. Rivers were marked well. Presence of the big number of details after the use of Canny operator in some cases could bring some issues during further steps, so additional filtering may be applied. In proposed method number of details less than after Canny operator and this could be useful. Later, selected edges could be used in segmentation and object detection algorithms [18, 19].

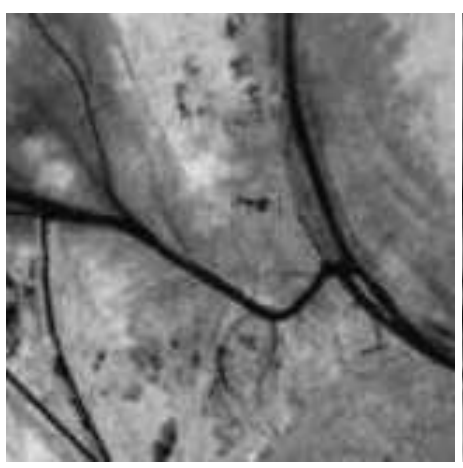

(a) source $f=184$

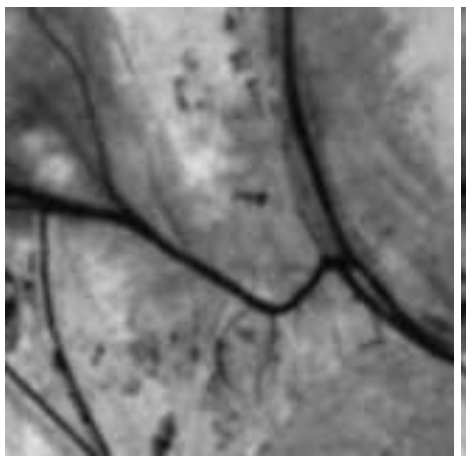

(d) avg. $D_{4} f=188$

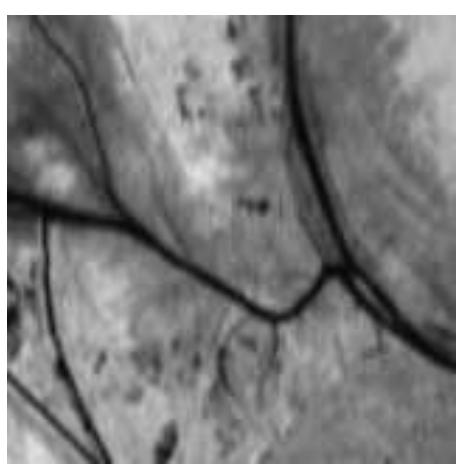

(b) avg. hor. $f=182$

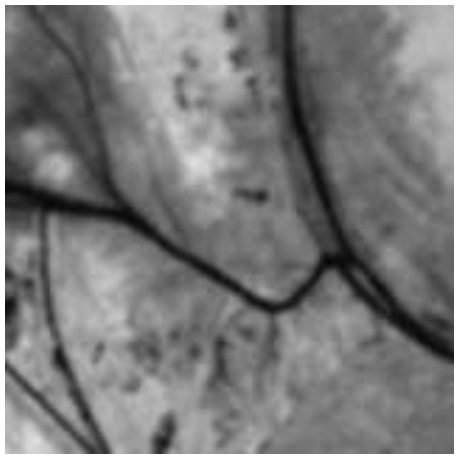

(e) avg. $D_{8} f=190$

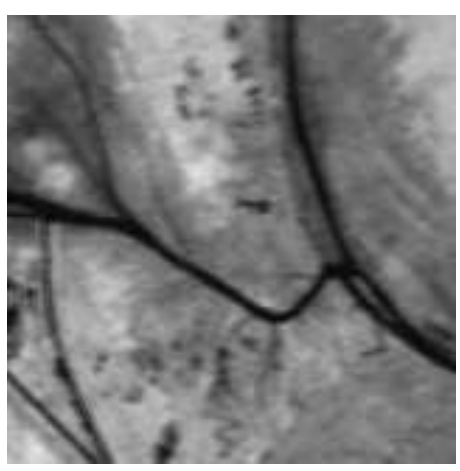

(c) avg. vert. $f=195$

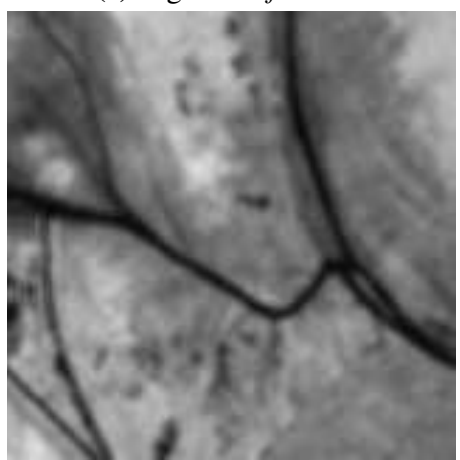

(f) avg. $D_{d} f=192$

Fig. 5. Remote sensing image of a river. Intensity level of the pixel $f=F(70,55)$ during different approaches to $I_{c}$ calculation.

Table 1. Intensities of pixels and according values of membership functions

\begin{tabular}{|c|c|c|}
\hline Image & $F(70,55)$ & $\pi(F(70,55))$ \\
\hline Source & 184 & 1 \\
\hline Avg. hor. & 182 & 0.9911 \\
\hline Avg. vert. & 195 & 0.7311 \\
\hline Avg. $D_{4}$ & 188 & 0.9644 \\
\hline Avg. $D_{8}$ & 190 & 0.92 \\
\hline Avg. $D_{d}$ & 192 & 0.8578 \\
\hline
\end{tabular}

\section{Conclusion}

Proposed algorithm showed their applicability in edge detection task during image processing. Main feature is the use of fuzzy image representation based on fuzzy pixels. This approach is very perspective because it saves the uncertainty much better rather other existing algorithms. In the future this model could be extended to type- 2 and higher fuzzy sets and also to other kinds of fuzzy sets. 


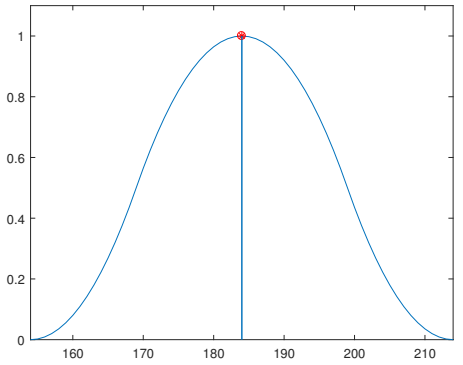

(a) source $\mu=1$

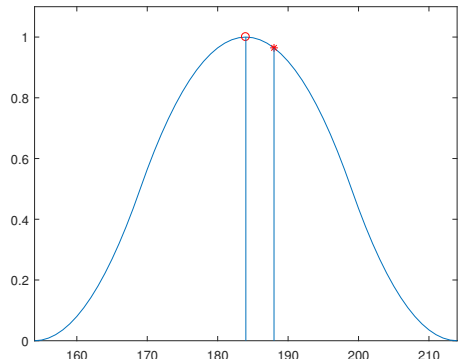

(d) avg. $D_{4} \mu=0.9644$

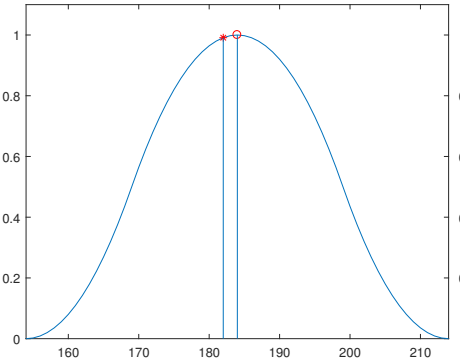

(b) avg. hor. $\mu=0.9911$

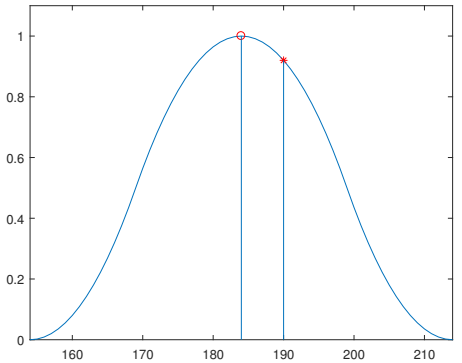

(e) avg. $D_{8} \mu=0.92$

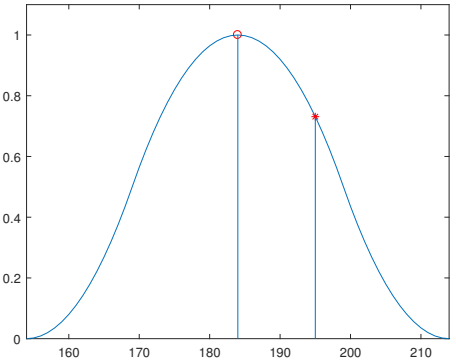

(c) avg. vert. $\mu=0.7311$

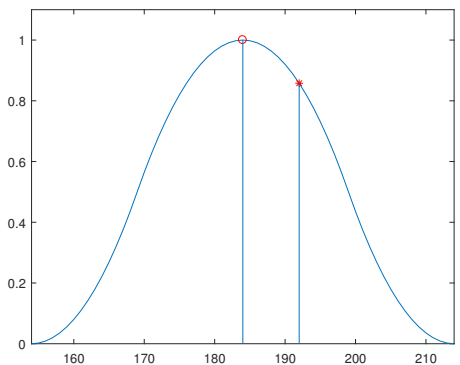

(f) avg. $D_{d} \mu=0.8578$

Fig. 6. Value of membership function $\mu=\mu(F(70,55))$ during different approaches to $I_{c}$ calculation.

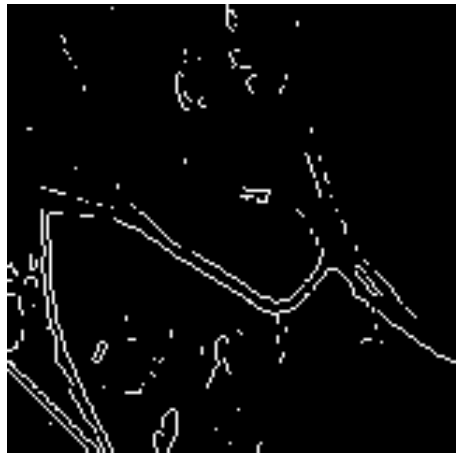

(a) Sobel operator

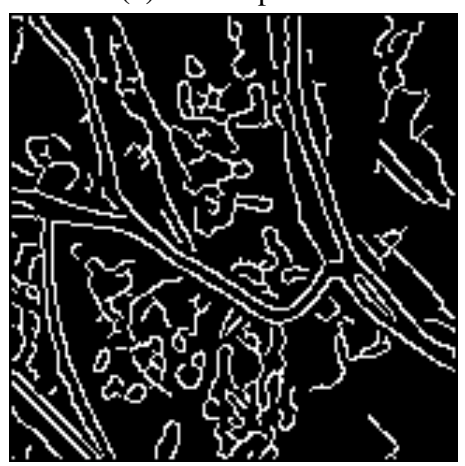

(d) Canny operator

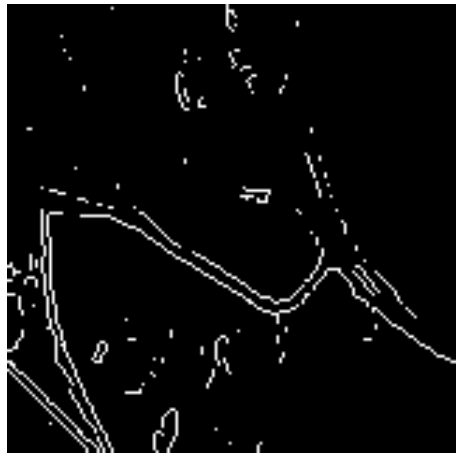

(b) Prewitt operator

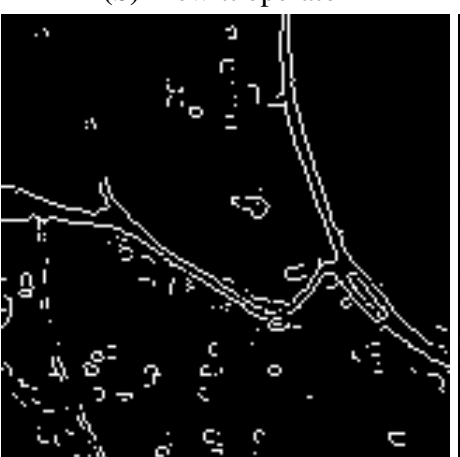

(e)

our method (based on Sobel operator)

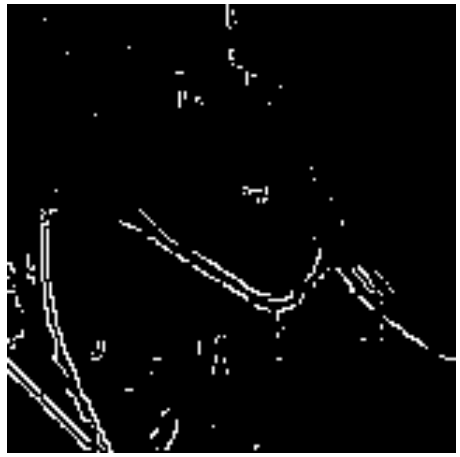

(c) Roberts operator

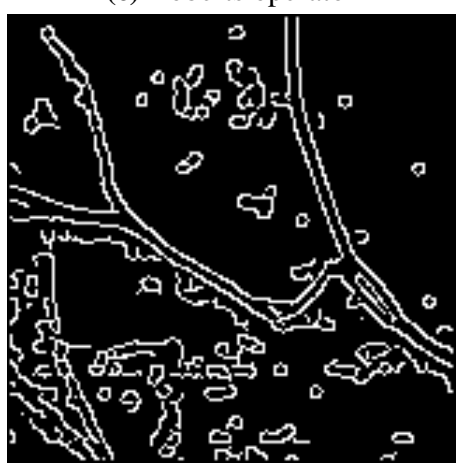

(f)

our method (based on Canny operator)

Fig. 7. Application of different edge detection operators. 


\section{References}

[1] Canny, J. A computational approach to edge detection / John Canny // Pattern Analysis and Machine Intelligence, IEEE Transactions on. — 1986. -11. - Vol. PAMI-8, no. 6. - P. 679-698.

[2] Kanopoulos, N. Design of an image edge detection filter using the sobel operator / N. Kanopoulos, N. Vasanthavada, R.L. Baker // IEEE Journal of Solid-State Circuits. - 1988. - apr. - Vol. 23, no. 2. - P. 358-367.

[3] Haralick, R. M. Digital step edges from zero crossing of second directional derivatives / Robert M. Haralick // IEEE Transactions on Pattern Analysis and Machine Intelligence. - 1984. - jan. - Vol. PAMI-6, no. 1. - P. 58-68.

[4] Kuo, Y.-H. A new fuzzy edge detection method for image enhancement / Yau-Hwang Kuo, Chang-Shing Lee, Chao-Chin Liu // Proceedings of 6th International Fuzzy Systems Conference. - [S. 1.] : Institute of Electrical and Electronics Engineers (IEEE), 1997.

[5] El-Khamy, S. A modified fuzzy sobel edge detector / S.E. El-Khamy, M. Lotfy, N. El-Yamany // Proceedings of the Seventeenth National Radio Science Conference. 17th NRSC 2000 (IEEE Cat. No.00EX396). - [S. 1.] : Institute of Electrical and Electronics Engineers (IEEE), 2002.

[6] Melin, P. An improved method for edge detection based on interval type-2 fuzzy logic / Patricia Melin, Olivia Mendoza, Oscar Castillo // Expert Systems with Applications. — 2010. — dec. - Vol. 37, no. 12. - P. 8527-8535.

[7] Becerikli, Y. A new fuzzy approach for edge detection / Yasar Becerikli, Tayfun M. Karan // Computational Intelligence and Bioinspired Systems. — [S. 1.] : Springer Nature, 2005. - P. 943-951.

[8] A novel fuzzy ant system for edge detection / Om Prakash Verma, Madasu Hanmandlu, Ashish Kumar Sultania, Dhruv // 2010 IEEE/ACIS 9th International Conference on Computer and Information Science. - [S. 1.] : Institute of Electrical and Electronics Engineers (IEEE), 2010. —aug.

[9] $\mathrm{Hu}, \mathrm{L}$. A high performance edge detector based on fuzzy inference rules / Liming Hu, H.D. Cheng, Ming Zhang // Information Sciences. — 2007. —nov. - Vol. 177, no. 21. - P. 4768-4784.

[10] Russo, F. Edge detection in noisy images using fuzzy reasoning / F. Russo // IMTC/98 Conference Proceedings. IEEE Instrumentation and Measurement Technology Conference. Where Instrumentation is Going (Cat. No.98CH36222). — [S. 1.] : Institute of Electrical and Electronics Engineers (IEEE), 1998.

[11] Interval type-2 fuzzy sets constructed from several membership functions: Application to the fuzzy thresholding algorithm / Miguel Pagola, Carlos LopezMolina, Javier Fernandez [et al.] // IEEE Transactions on Fuzzy Systems. — 2013. —apr. — Vol. 21, no. 2. —P. $230-244$.

[12] Fuzzy Techniques in Image Processing / Ed. by Etienne E. Kerre, Mike Nachtegael. — [S. 1.] : Physica-Verlag HD, 2000.

[13] Edge-detection method for image processing based on generalized type-2 fuzzy logic / Patricia Melin, Claudia I. Gonzalez, Juan R. Castro [et al.] // IEEE Transactions on Fuzzy Systems. - 2014. — dec. — Vol. 22, no. 6. - P. 1515-1525.

[14] An improved sobel edge detection method based on generalized type-2 fuzzy logic / Claudia I. Gonzalez, Patricia Melin, Juan R. Castro [et al.] // Soft Computing. - 2014. - dec. - Vol. 20, no. 2. - P. 773-784.

[15] Molodtsov, D. Soft set theoryfirst results / D. Molodtsov // Computers \& Mathematics with Applications. — 1999. — Vol. 37, no. 4. — P. 19 - 31. — URL: http://www.sciencedirect.com/science/article/pii/S0898122199000565.

[16] Pawlak, Z. Rough sets / Zdzislaw Pawlak // International Journal of Computer and Information Sciences. — 1982. —oct. — Vol. 11, no. 5. — P. 341-356. - URL: http://dx.doi.org/10.1007/BF01001956.

[17] Otsu, N. A threshold selection method from gray-level histograms / N Otsu // IEEE Trans. Sys., Man., Cyber. — 1979. — Vol. 9. — P. 62-66.

[18] Privezentsev, D. G. Use of characteristic image segments in tasks of digital image processing / Denis G. Privezentsev, Arkady L. Zhiznyakov // 2015 International Conference "Stability and Control Processes" in Memory of V.I. Zubov (SCP). — [S. 1.] : Institute of Electrical and Electronics Engineers (IEEE), 2015. —oct.

[19] Zhiznyakov, A. L. Using fractal features of digital images for the detection of surface defects / A. L. Zhiznyakov, D. G. Privezentsev, A. A. Zakharov // Pattern Recognition and Image Analysis. - 2015. - jan. - Vol. 25, no. 1. - P. 122-131. 